\title{
EDITORIAL
}

\section{Seeking the right targets: gene therapy advances in pulmonary arterial hypertension}

\author{
R.D. Machado
}

$\mathbf{P}$ ulmonary arterial hypertension (PAH) is a grave vascular disorder characterised by the obstruction and occlusion of the small pulmonary arteries, primarily through the abnormal proliferation of the endothelial and smooth muscle layers. A clinical diagnosis of pulmonary hypertension $(\mathrm{PH})$ is made when the mean pulmonary arterial pressure $\left(\bar{P}_{\mathrm{pa}}\right)$ is $\geqslant 25 \mathrm{mmHg}$ at rest. An additional criterion to differentiate PAH from other forms of $\mathrm{PH}$ requires that pulmonary arterial wedge pressure be $\geqslant 15 \mathrm{mmHg}[1,2]$. The disease is typically fatal due to right heart failure as a result of the pathological increases in pulmonary vascular resistance. While the majority of the patient population develop spontaneous or idiopathic PAH and PAH associated with other disorders, for example, HIV infection, connective tissue disease or exposure to risk factors such as appetite suppressants containing dex/fenfluramine (associated $\mathrm{PAH}$ ), some 10\% display familial transmission. In families, $\mathrm{PAH}$ is an autosomal dominant trait with a sex bias favouring females and penetrance as low as $20 \%$, features that suggest that, while a Mendelian condition, PAH displays features of complex disease [1, 2].

Traditional positional cloning approaches identified the causative gene for familial PAH as BMPR2, encoding a type-II receptor of the transforming growth factor (TGF)- $\beta$ signalling family $[3,4]$. Currently, deleterious germ-line mutations have been detected in $\sim 70 \%$ of families analysed and $25 \%$ of idiopathic cases $[5,6]$. The recent re-classification of mutation carriers with or without a family history as heritable PAH (HPAH), at the 4th World symposium on Pulmonary Hypertension (Dana Point, CA, USA) in 2008, indicates the growing recognition of the central importance of $B M P R 2$ dysfunction in pathogenesis [7]. The majority of $B M P R 2$ mutation in HPAH predicts premature truncation of the transcript and, thus, haploinsufficiency is most probably the predominant molecular mechanism of disease $[6,8]$. Interestingly, mutation carriers exhibit progressive loss of BMPR-II levels in the lung, substantially below that predicted by haploinsufficiency, which is suggestive of unexplained loss of the wild-type protein. Moreover, even in the absence of BMPR2 mutation, patients and experimental models exhibit a significant reduction in BMPR-II protein levels through mechanisms that remain to be elucidated [9-11]. BMPR-II transduces signal in concert with a type-I partner, which may be BMPR1A/B or ALK1, via a phosphorylation relay mediated by the SMAD

CORRESPONDENCE: R.D. Machado, King's College London, Dept of Medical and Molecular Genetics, School of Medicine, Guy's Hospital, London, SE1 9RT, UK. E-mail: rajiv.machado@genetics.kcl.ac.uk family $[12,13]$. The requirement of these molecules in maintenance of the pulmonary vascular bed is emphasised by the identification of rare PAH-predisposing mutations in several members of the pathway, in particular ALK1 and the type-III receptor endoglin, although recent data also indicates the involvement of members of the SMAD family [14-18]. While the precise mechanisms driving susceptibility to $\mathrm{PAH}$ remain unclear, a notable recent finding has been the apparent inverse correlation between BMPR-II and TGF- $\beta$ levels, suggesting that the exuberant proliferation of endothelial and smooth muscle cells observed in the pulmonary vasculature may be reliant on heightened TGF- $\beta$ signalling [19].

In the past, PAH was characterised by high mortality and morbidity, with death typically occurring 3 yrs post-diagnosis. The last two decades have seen a significant improvement in treatment options available to PAH patients yet these pharmacological agents remain merely palliative and come with several caveats. Acute vasodilator therapy, namely in the form of calcium channel blockers, whilst highly efficacious is an option in a small proportion of patients due to the fact that upon vasodilator challenge only very few demonstrate vasoreactivity. Insights into the pathophysiology of PAH have implicated three pathways in the development and progression of disease: the prostacyclin, nitric oxide and endothelin pathways. Each of these have since become therapeutic targets and subsequent treatments have been developed. Prostacylin analogues have been shown to improve quality of life and survival but some require continuous infusion and can be prohibitively expensive. The advent of prostacyclin analogues that may be administered by inhalation is a recent and exciting development but longterm effects on survival are unclear. Endothelin receptor antagonists and phosphodiesterase inhibitors have demonstrated modest clinical improvement in patients but, due to the progressive nature of $\mathrm{PAH}$, these newer agents often have to be administered in combination, with the potential for the development of unpleasant side-effects. It is an unfortunate truth that while the present therapies represent a major improvement on the treatments of old, in a great many patients responsiveness decreases and, in the absence of lung transplantation, premature death does occur [20, 21]. Based on contemporary data derived from the French Network on Pulmonary Hypertension and the Registry to Evaluate Early and Long-term Pulmonary Arterial Hypertension (REVEAL) based in the USA, 3-yr survival rates in patients are still somewhat underwhelming [22-24]. Thus, the need for novel, yet more effective treatment options and protocols is still a pressing issue and constitutes the ultimate focus of the majority of PAH 
research centres worldwide. The ideal scenario, with respect to current treatments, is the development of an intervention that would not merely ameliorate symptoms but, instead, drive the permanent reversal of pathological features such as vascular remodelling and vasoconstriction in patient lungs.

Although explored in the treatment of $\mathrm{PAH}$, gene-based therapy has not been substantively pursued, perhaps due to the inherent limitations of the technology at the present time, which include efficient vector design and delivery, potential risks of insertional mutagenesis, duration of effect and, in particular, precise targeting of the key cellular sites of disease.

The study by REYNOLDS et al. [25] in the current issue of the European Respiratory Journal addresses a number of these crucial and long-standing problems and may, indeed, represent a first step in the development of a next generation of treatment options in PAH. Using two experimental rat models, namely chronic hypoxia and monocrotaline (MCT)-induced $\mathrm{PH}$, the authors demonstrate the development of haemodynamic and histopathological features of $\mathrm{PH}$ with no evidence of systemic involvement. Both models exhibited depressed levels of BMPR-II recapitulating the hallmark molecular defect observed in human disease. Employing an adenoviral $B M P R 2$ gene delivery vector targeted to the pulmonary endothelium via linkage to a bispecific antibody to the angiotensinconverting enzyme, they detected receptor expression in the pulmonary arterioles and alveolar capillaries but not in cardiac sections, implying an improvement in the degree of tissue selectivity. The subsequent use of this resource in analysing the impact on $\mathrm{PH}$ reveals some important findings. Upon vector delivery, significant improvements in pulmonary haemodynamics were observed in the MCT model but were less evident in the hypoxic animals, notably in $\bar{P}_{\text {pa }}$ and right ventricular systolic pressure measurements, although a trend toward recovery was observed. Vascular muscularisation was reduced in both models upon treatment allied to a reduction in abnormal proliferation of the endothelial and smooth muscle layers. A common feature of human $\mathrm{PAH}$ is the abnormal and probably pathological upregulation of TGF- $\beta$ in the lung, a defect shared by the MCT-treated but not the hypoxia-induced rat model. Restoration of BMPR-II levels through adenoviral delivery markedly diminished the TGF- $\beta$ response. The authors determined in vitro, in human pulmonary microvascular endothelial cells, that restitution of BMPR-II attenuated TGF- $\beta$-induced endothelial-mesenchymal transition which, while interesting and worth pursuing, is at the present juncture of uncertain mechanistic import in the development of PAH. The fundamental finding of this study that reconstitution of BMPR-II expression has a beneficial effect in $\mathrm{PH}$ arising from different pathological backgrounds is of considerable interest, indicating as it does that a loss of BMPR-II activity in the pulmonary endothelium is the common link between different forms of $\mathrm{PH}$ and fundamental to disease progression.

Whether these data represent the beginning of an imminent new dawn of PAH treatments is difficult to gauge. As the authors candidly point out, in translating this preliminary research to clinic many hurdles lie ahead, not least in the development of next-generation vectors capable of maintaining long-term gene expression. At present, gene expression runs for only a number of weeks and repeat administration of vector is likely to be compromised by the host immune response. It is unclear whether a single, short-lived treatment would be sufficient in producing a sustained beneficial outcome in patients and this has not been addressed here. It is equally necessary to fully elucidate the molecular impact of BMPR-II expression restoration on downstream pathways dysregulated in human disease, the aetiology of which is likely to be very distinct from experimental rat models. What this study certainly does accomplish is to establish convincing and encouraging biological evidence that increasing BMPR-II levels, in the right tissue type, have positive therapeutic consequences for multiple forms of experimental $\mathrm{PH}$. Now, it is necessary for the technology the authors employ in this work to be suitably advanced to the level required for broad clinical utility, for translation to be achieved.

\section{STATEMENT OF INTEREST}

None declared.

\section{REFERENCES}

1 Galiè N, Hoeper MM, Humbert M, et al. Guidelines for the diagnosis and treatment of pulmonary hypertension. Eur Respir J 2009; 34: 1219-1263.

2 Austin ED, Loyd JE, Phillips JA 3rd. Genetics of pulmonary arterial hypertension. Semin Respir Crit Care Med 2009; 30: 386-398.

3 Lane KB, Machado RD, Pauciulo MW, et al. Heterozygous germline mutations in $B M P R 2$, encoding a TGF- $\beta$ receptor, cause familial primary pulmonary hypertension. Nat Genet 2000; 26: 81-84.

4 Deng Z, Morse JH, Slager SL, et al. Familial primary pulmonary hypertension (gene PPH1) is caused by mutations in the bone morphogenetic protein receptor-II gene. Am J Hum Genet 2000; 67: 737-744.

5 Machado RD, Eickelberg O, Elliott CG, et al. Genetics and genomics of pulmonary arterial hypertension. J Am Coll Cardiol 2009; 54: Suppl. 1, S32-S42.

6 Aldred MA, Vijayakrishnan J, James V, et al. BMPR2 gene rearrangements account for a significant proportion of mutations in familial and idiopathic pulmonary arterial hypertension. Hum Mutat 2006; 27: 212-213.

7 Simonneau G, Robbins IM, Beghetti M, et al. Updated clinical classification of pulmonary hypertension. J Am Coll Cardiol 2009; 54: Suppl. 1, S43-S54.

8 Machado RD, Pauciulo MW, Thomson JR, et al. BMPR2 haploinsufficiency as the inherited molecular mechanism for primary pulmonary hypertension. Am J Hum Genet 2001; 68: 92-102.

9 Atkinson C, Stewart S, Upton PD, et al. Primary pulmonary hypertension is associated with reduced pulmonary vascular expression of type II bone morphogenetic protein receptor. Circulation 2002; 105: 1672-1678.

10 Takahashi H, Goto N, Kojima Y, et al. Downregulation of type II bone morphogenetic protein receptor in hypoxic pulmonary hypertension. Am J Physiol Lung Cell Mol Physiol 2006; 290: L450-L458.

11 Morty RE, Nejman B, Kwapiszewska G, et al. Dysregulated bone morphogenetic protein signaling in monocrotaline-induced pulmonary arterial hypertension. Arterioscler Thromb Vasc Biol 2007; 27: 1072-1078.

12 Miyazono K, Kamiya Y, Morikawa M. Bone morphogenetic protein receptors and signal transduction. J Biochem 2010; 147: 35-51.

13 Upton PD, Davies RJ, Trembath RC, et al. Bone morphogenetic protein (BMP) and activin type II receptors balance BMP9 signals mediated by activin receptor-like kinase- 1 in human pulmonary artery endothelial cells. J Biol Chem 2009; 284: 15794-15804.

14 Harrison RE, Flanagan JA, Sankelo M, et al. Molecular and functional analysis identifies ALK-1 as the predominant cause of 
pulmonary hypertension related to hereditary haemorrhagic telangiectasia. J Med Genet 2003; 40: 865-871.

15 Girerd B, Montani D, Coulet F, et al. Clinical outcomes of pulmonary arterial hypertension in patients carrying an ACVRL1 (ALK1) mutation. Am J Respir Crit Care Med 2010; 181: 851-861.

16 Fujiwara M, Yagi $H$, Matsuoka R, et al. Implications of mutations of activin receptor-like kinase 1 gene (ALK1) in addition to bone morphogenetic protein receptor II gene (BMPR2) in children with pulmonary arterial hypertension. Circ J 2008; 72: 127-133.

17 Shintani M, Yagi H, Nakayama T, et al. A new nonsense mutation of SMAD8 associated with pulmonary arterial hypertension. J Med Genet 2009; 46: 331-337.

18 Nasim MT, Ogo T, Ahmed M, et al. Molecular genetic characterization of SMAD signaling molecules in pulmonary arterial hypertension. Hum Mutat 2011; 32: 1385-1389.

19 Long L, Crosby A, Yang X, et al. Altered bone morphogenetic protein and transforming growth factor- $\beta$ signaling in rat models of pulmonary hypertension: potential for activin receptor-like kinase-5 inhibition in prevention and progression of disease. Circulation 2009; 119: 566-576.
20 Agarwal R, Gomberg-Maitland M. Current therapeutics and practical management strategies for pulmonary arterial hypertension. Am Heart J 2011; 162: 201-213.

21 Corris PA. Alternatives to lung transplantation: treatment of pulmonary arterial hypertension. Clin Chest Med 2011; 32: 399-410.

22 Humbert M, Sitbon O, Chaouat A, et al. Survival in patients with idiopathic, familial, and anorexigen-associated pulmonary arterial hypertension in the modern management era. Circulation 2010; 122: $156-163$.

23 Benza RL, Miller DP, Gomberg-Maitland M, et al. Predicting survival in pulmonary arterial hypertension: insights from the Registry to Evaluate Early and Long-Term Pulmonary Arterial Hypertension Disease Management (REVEAL). Circulation 2010; 122: $164-172$.

24 Humbert M, Sitbon O, Yaïci A, et al. Survival in incident and prevalent cohorts of patients with pulmonary arterial hypertension. Eur Respir J 2010; 36: 549-555.

25 Reynolds AM, Holmes MD, Danilov SM, et al. Targeted gene delivery of BMPR2 attenuates pulmonary hypertension. Eur Respir J 2012; 39: 329-343. 